\title{
Diffractive Optical Elements with a Large Angle of Operation Recorded in Acrylamide Based Photopolymer on Flexible Substrates
}

\author{
Hoda Akbari, ${ }^{1}$ Izabela Naydenova, ${ }^{1}$ Lina Persechini, ${ }^{2}$ Sean M. Garner, ${ }^{3}$ \\ Pat Cimo, ${ }^{3}$ and Suzanne Martin ${ }^{1}$ \\ ${ }^{1}$ Centre for Industrial and Engineering Optics, School of Physics, Dublin Institute of Technology, Kevin Street, Dublin 8, Ireland \\ ${ }^{2}$ Optoelectronics Research Centre, University of Southampton, Southampton SO17 1BJ, UK \\ ${ }^{3}$ Corning Incorporated, One Riverfront Plaza, Corning, NY 1431, USA
}

Correspondence should be addressed to Suzanne Martin; suzanne.martin@dit.ie

Received 26 August 2014; Revised 4 November 2014; Accepted 4 November 2014; Published 11 December 2014

Academic Editor: Sergi Gallego

Copyright (C) 2014 Hoda Akbari et al. This is an open access article distributed under the Creative Commons Attribution License, which permits unrestricted use, distribution, and reproduction in any medium, provided the original work is properly cited.

\begin{abstract}
A holographic device characterised by a large angular range of operation is under development. The aim of this study is to increase the angular working range of the diffractive lens by stacking three layers of high efficiency optical elements on top of each other so that light is collected (and focussed) from a broader range of angles. The angular range of each individual lens element is important, and work has already been done in an acrylamide-based photosensitive polymer to broaden the angular range of individual elements using holographic recording at a low spatial frequency. This paper reports new results on the angular selectivity of stacked diffractive lenses. A working range of $12^{\circ}$ is achieved. The diffractive focussing elements were recorded holographically with a central spatial frequency of $300 \mathrm{l} / \mathrm{mm}$ using exposure energy of $60 \mathrm{~mJ} / \mathrm{cm}^{2}$ at a range of recording angles. At this spatial frequency with layers of thickness $50 \pm 5 \mu \mathrm{m}$, a diffraction efficiency of $80 \%$ and $50 \%$ was achieved in the single lens element and combined device, respectively. The optical recording process and the properties of the multilayer structure are described and discussed. Holographic recording of a single lens element is also successfully demonstrated on a flexible glass substrate (Corning(R) Willow(R) Glass) for the first time.
\end{abstract}

\section{Introduction}

Photopolymers are fast becoming one of the most popular recording media for holographic applications for a variety of reasons; they have excellent holographic characteristics, such as high refractive index modulation, large dynamic range, good light sensitivity, real time image development, high optical quality, and low cost. Photopolymer materials have been investigated for use in a number of holographic applications [1-8]. Recent work proposes stacking of low spatial frequency transmission holograms [9] and multiplexing of high spatial frequency transmission Holograms [10] in different photopolymers as promising approaches for tracking-free sunlight redirecting devices.

An ideal holographic recording material should have the following properties: high sensitivity to available commercial wavelengths, high spatial resolution for improved quality recording, linear response to recording intensities, and low noise, that is, a fine grain structure to reduce scatter effects. In applications where low angular selectivity is necessary, the ability to record at lower spatial frequencies is also beneficial. Considerations regarding material flexibility, substrate flexibility, substrate thickness, substrate optical properties, and good adhesion are also of significance, particularly when stacking layers.

Over the years a number of other researchers have studied the response of the low spatial frequency gratings in photopolymer materials to be used in different applications. For example, Tarjanyi et al. [11] reported on the response of photopolymer material at low spatial frequency and different recording intensities and Pascual et al. [12] have investigated the capability of a photopolymer recording material for mass 
production of computer generated gratings at low spatial frequencies.

In the work presented here a self-developing acrylamidebased photopolymer has been used as the photosensitive medium [13-18]. The capability of this material to record at low spatial frequencies has been attributed to the relatively high permeability of the polymer matrix and fast monomer diffusion, which allows for the recording of high diffraction efficiency gratings even in the low spatial frequency regime [19]. Such low spatial frequencies are preferable when devices with larger acceptance angle (broad angular and wavelength selectivity operation ranges) are sought. Keeping the spatial frequency low ensures that the angular selectivity of each individual grating/lens is low so that the range of angles accepted by each individual grating is maximized and the number of gratings needed in the combined device is minimized. In this work we present low spatial frequency photopolymer holographic lenses stacked together in order to increase the angular range of the focussing device.

\section{Theoretical Background}

For a given material, diffraction efficiency depends directly on the thickness of the holograms, but the angular and wavelength selectivity depends on both thickness and the spatial frequency of the grating. In agreement with volume holographic grating theory [20], a previous study demonstrated that gratings and lenses recorded on thinner layers at low spatial frequencies have a much greater acceptance angle [9]. For example, the acceptance angle for gratings recorded in $50 \mu \mathrm{m}$ thick layers at a spatial frequency of $10001 / \mathrm{mm}$ is about $1^{\circ}$, whereas gratings recorded at spatial frequency of $300 \mathrm{l} / \mathrm{mm}$ show an acceptance angle of approximately $3^{\circ}$. Multiplexing many gratings in one layer has been shown to work well [4]; however the diffraction efficiency of each grating decreases with the number of gratings recorded due to the limited dynamic range of the photopolymer.

In order to increase the angular range further without reducing the diffraction efficiency of each element, a number of holograms can be stacked by laminating them together using flexible substrates. The use of flexible substrates (such as plastic and flexible glass [21]) has significant advantages over conventional thicker glass as is flexible, conforms to the required shape, and has a reduced weight as well as thickness, implying lower losses for stacking devices. Examples of stacked displays on flexible glass substrates have previously been demonstrated [22]. Use of flexible substrates also enables high-volume continuous manufacturing methods such as roll-to-roll device fabrication [23].

In this study the holographic elements used are focussing elements, also referred to as diffractive optical element (DOE) lenses. DOE lenses are made by interfering a spherically focussed beam with a reference beam (collimated) and arranging the photopolymer layer at the overlap as described in Section 3.3. The photopolymer material responds by producing a local variation in refractive index that records the interference fringe planes formed as the spherical and plane waves interfere. Controlling the interference pattern allows

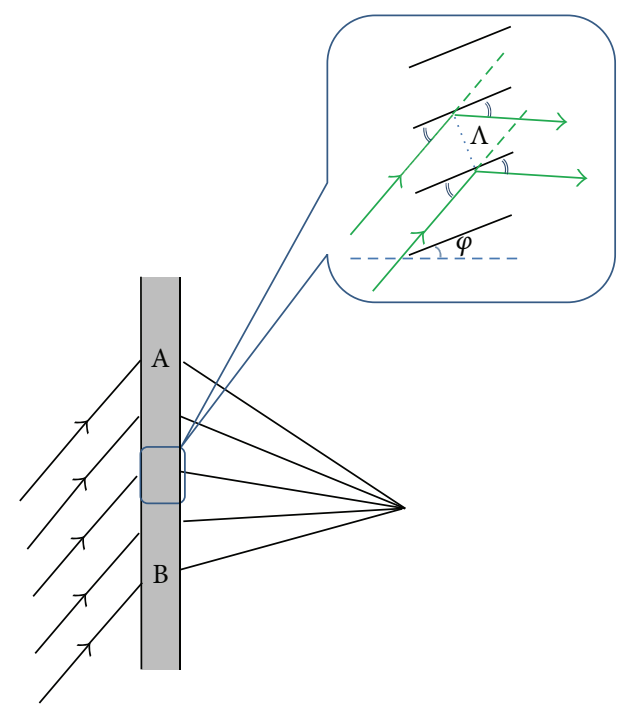

FIGURE 1: Schematic of the diffractive optical elements (DOEs) which redirects the incident light to an off-axis focus point, with inset showing typical fringe structure. Where $\Lambda$ is the fringe spacing and $\varphi$ is the angle between the fringes and the normal to the plane of the recording medium.

control of the diffraction properties of the element recorded. The performance of the DOEs is characterised in terms of diffraction efficiency and angular selectivity.

In this approach each element is designed to focus an off-axis collimated incident beam to a point behind the holographic element, as shown below. For this study, three off-axis focussing elements are then stacked together, each designed for a different angle of incidence, so that when combined, the stack is capable of focusing light incident from a broader range of angles. This arrangement of layers also requires a careful control of their thickness so that each focusing elements has the expected FWHM working range.

Figure 1 is a schematic diagram showing how the individual DOE redirects the incident light. The DOE is a complex diffractive element which can be thought of as a series of slanted gratings that redirect the incident light towards the focal point. The spatial frequency and slant angle of the grating planes vary across the DOE. For example, the spatial frequency of the grating at point $\mathrm{A}$ in the diagram will be much greater than the spatial frequency at point $B$, but the slant angle of the grating planes will be greater at B than A. The material used must be capable of recording the desired range of spatial frequencies.

In this study, in order to increase the operation angle of the device three individual layers of high efficiency DOEs are recorded at three different positions of the recording material with respect to the interference pattern. After recording they are laminated on top of each other without any air gap in order to reduce reflection losses.

Since they can be considered thick holograms, the maximum diffraction efficiency is achieved when the DOEs are probed at the correct reconstruction angle. Each grating element within the DOE should be illuminated at the Bragg angle for that grating. This is achieved using a collimated 
TABLE 1: Concentrations of the photopolymer composition.

\begin{tabular}{lc}
\hline Components & Amount \\
\hline Acrylamide & $11 \mathrm{mmol}$ \\
Methylenebisacrylamide & $1.3 \mathrm{mmol}$ \\
Polyvinyl alcohol $(10 \% \mathrm{wt} / \mathrm{v}$ stock $)$ & $17.5 \mathrm{~mL}$ \\
Triethanolamine & $15 \mathrm{mmol}$ \\
Erythrosine B dye $(0.11 \% \mathrm{wt} / \mathrm{v})$ & $5 \mu \mathrm{mol}$ \\
\hline
\end{tabular}

beam in both recording and reconstruction. For an unslanted transmission grating, the Bragg condition is given by

$$
m \lambda=2 \Lambda \sin \theta
$$

where $m$ is the diffraction order (for thick holograms $m=$ 1), $\theta$ is the Bragg angle defined as the angle that the incident beam makes with the fringe plane in the recording medium, $\lambda$ is the wavelength of the recording beam, and $\Lambda$ is the fringe spacing.

The recorded DOEs in this study are considered as thick holograms [20,24-26], because $Q \geq 1$, where parameter $Q$ is defined by

$$
Q=\frac{2 \pi \lambda d}{n_{0} \Lambda^{2}},
$$

where $d$ is the thickness of the recording medium, $n_{0}$ is the average refractive index of the recording material, and $\Lambda$ is previously defined.

In this study these parameters correspond to a $Q$ factor of about 10 . This value was estimated by taking the spatial frequency of $300 \mathrm{l} / \mathrm{mm}$ at the centre of the lens and the photosensitive layer thickness of $50 \pm 5 \mu \mathrm{m}$.

Kogelnik predicts the relationship between incident angle and diffraction efficiency in thick gratings, for specific conditions [20]. For gratings that are not overmodulated there is a maximum at the Bragg angle and the width of the peak depends on grating thickness and spatial frequency. The lenses presented here are characterised by measuring the diffraction efficiency over a range of angles in order to determine this angular selectivity. When interpreting the results it should be borne in mind that for such DOEs a range of spatial frequencies and slant angles are always present.

\section{Experimental}

3.1. Photopolymer Solution Preparation. The material used in this research is a self-developing acrylamide-based watersoluble photopolymer as previously described [13]. The composition of this material is acrylamide and methylenebisacrylamide monomers, triethanolamine as an initiator, a polyvinyl alcohol binder, and an erythrosine $B$ as a sensitizer. The average refractive index of the fabricated photopolymer layer is approximately $n_{0}=1.50$ [27].

Table 1 shows the component concentrations of the photopolymer solution used to obtain layers of $50 \pm 5 \mu \mathrm{m}$ thick, as confirmed by white light interferometry.

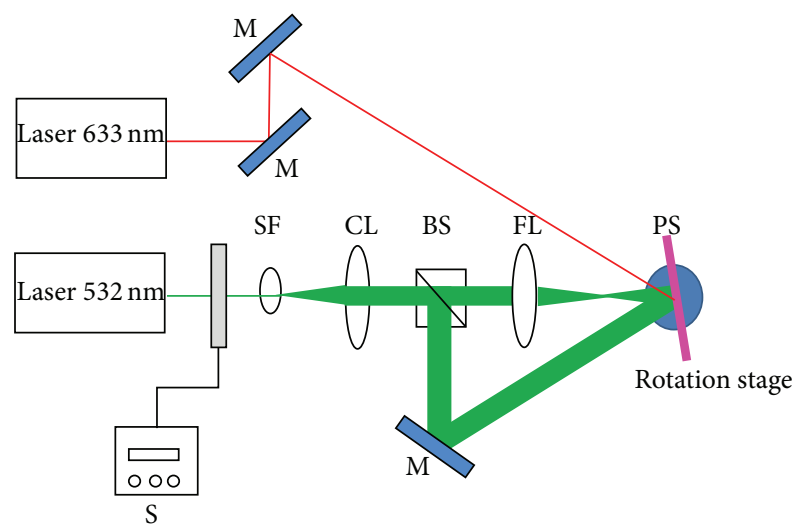

FIGURE 2: Experimental set-up for recording focussing transmission holograms: S: shutter, CL: collimating lens, BS: beam splitter, SF: spatial filter, M: mirror, FL: focusing lens, and PS: photopolymer sample.

3.2. Layer Preparation. $0.5 \mathrm{~mL}$ of the photopolymer solution was spread evenly using the gravity settling method on a $25 \times$ $75 \mathrm{~mm}^{2}$ flexible plastic (Bayer's Makrofol) or glass substrate and then placed on a levelled surface and allowed to dry for 18-24 hours in darkness under normal laboratory conditions $\left(20-25^{\circ} \mathrm{C}, 40-60 \% \mathrm{RH}\right)$.

3.3. Holographic Set-Up. DOEs were recorded in a twobeam holographic optical set-up (Figure 2) using a vertically polarized $\mathrm{Nd}: \mathrm{YVO}_{4}$ laser (532 nm), and a Helium-Neon laser $(\mathrm{He}-\mathrm{Ne})$ at $633 \mathrm{~nm}$ was used as a probe beam. Both the recording beams and the probe beam are vertically polarized.

The intensity of the recording beams was set by a variable neutral density filter to $1 \mathrm{~mW} / \mathrm{cm}^{2}$. Previous studies have shown this intensity to be optimum for recording at a spatial frequency of $3001 / \mathrm{mm}$ [9]. A spatial frequency of 300 lines/mm was obtained by adjusting the interbeam angle to $9^{\circ}$. The exposure time was kept constant at $60 \mathrm{~s}$; thus an exposure energy of $60 \mathrm{~mJ} / \mathrm{cm}^{2}$ in a layer of thickness $50 \pm 5 \mu \mathrm{m}$ was achieved. A rotation stage was used to set the angle of the recording medium with respect to the recording beams, for each element. In order to characterize the diffracted intensity dependence on the incident angle of the probe beam, the grating was placed on a rotation stage (Newport, ESP 300). An optical power meter (Newport 1830C) recorded the intensity of the diffracted beam and the data was transferred to a computer via a data acquisition card. A LabVIEW program was used to control the experiment and to record the data.

The first step of this experiment involves the characterization of the Bragg selectivity curve for each layer individually. The Bragg curve can provide information about the grating/lens parameters such as efficiency, thickness, angular full width half $\max$ (FWHM), and refractive index modulation. The second step involves the study of the angular selectivity of the device after stacking three DOE lenses, recorded in individual layers, on top of each other. The distance from the photopolymer sample to the focal point of lens defines 
the focal length of the recorded DOE, where in this study the fabricated elements have a focal length of about $6 \mathrm{~cm}$. The focal length of fabricated DOEs can be varied by changing the distance between the focus point of the lens to the photopolymer recording material.

The diffraction efficiency of the recorded DOE lenses determines the efficiency of each element at redirecting light to the appropriate angle and is defined as

$$
\eta=\frac{I_{d}}{I_{\text {in }}} \times 100,
$$

where $I_{d}$ is diffracted beam intensity, $I_{\text {in }}$ is incident (probe beam) intensity, and $\eta$ is diffraction efficiency. Using the ratio of the intensity in the diffracted beam to the incident intensity means that reflection, absorption, scatter, and other losses in the substrates and photopolymer will lower the diffraction efficiency value, but it allows a more realistic estimate of the usefulness of the elements in collecting light.

A range of high efficiency DOE lenses were holographically recorded at three different positions of the recording material with respect to the interference pattern created by the recording beams. The central spatial frequency was relatively low $-300 \mathrm{l} / \mathrm{mm}$. The layers then were stacked on top of each other. Lamination of the photopolymer layer to the substrate of its immediate neighbour in the stack ensures good adhesion and no air gap. The key challenge is to control the working ranges of the individual holographic elements so that when laminated together the Bragg selectivity curves overlap sufficiently. In other words, as the angle of incidence changes and the first grating efficiency begins to drop the second should begin to rise.

The experiment was carried out by characterizing the angular response of the zero diffraction order and the first diffraction order of the recorded lenses before and after stacking. In this work, optical elements were aligned by using the grating/lens edge as a guide. Angular alignment depends on the effective lamination of each layer to the next and careful control of the recording angles for each diffractive element.

\section{Results and Discussion}

The following results show how diffraction efficiency varies with angle of incidence for individual holographic focussing elements, recorded in photopolymer on plastic substrates, as well as a combined stack of three elements. Successful recording is also demonstrated in photopolymer on flexible glass substrates using the same techniques.

4.1. Comparison of the Acceptance Angle of Diffractive Optical Elements before and after Stacking. The variation of the diffraction efficiency with angle of incidence in the zero and first diffraction orders for holographic lenses recorded at range of angles $\left(7^{\circ}, 10.5^{\circ}\right.$, and $\left.14^{\circ}\right)$ is shown in Figure 3. A diffraction efficiency of about $80 \%$ was observed. The FWHM is between 4.5 and $5^{\circ}$ for each lens element.

Figure 4 shows the variation of diffraction efficiency with angle of incidence for the zero and first-order diffraction of
TABLE 2: The comparison of transmittance and reflectance of three substrates.

\begin{tabular}{lccc}
\hline Substrate & Transmittance & Reflectance & Refractive index \\
\hline Standard glass & $90.1 \pm 0.6 \%$ & $6.8 \pm 0.5 \%$ & 1.5 \\
Plastic & $89.6 \pm 1.6 \%$ & $9.7 \pm 0.7 \%$ & 1.503 \\
Flexible glass & $91.9 \pm 0.7 \%$ & $7.2 \pm 0.9 \%$ & 1.504 \\
\hline
\end{tabular}

the stacked elements from Figure 3. It can be observed that the FWHM was increased to collect light from a working range of approximately $12^{\circ}$; however, losses in diffraction efficiency have occurred in the gratings after stacking as the diffraction efficiency has dropped to approximately $50 \%$. Even though the layers appear to laminate together exceptionally well this reduction in efficiency may be caused by cumulative losses (scattering and reflection) at the multiple layer interfaces, since there are six interfaces in total including substrates. The decrease in diffraction efficiency after stacking the layers may be improved by using a different substrate where the refractive index will be better matched and moving to a thinner more transparent substrate with reduced birefringence, haze, and scattering. Flexible glass, such as Willow Glass, could be the solution. Table 2 displays the measured optical properties of three substrates.

The transmittance and reflectance of the three substrates available have been compared by using a laser with wavelength of $633 \mathrm{~nm}$ incident at 10.5 degree on the substrate and the results are shown in Table 2. Each transmittance and reflectance value is an average of 8 readings. The refractive index of the plastic and flexible glass substrates was measured using an Abbe refractometer. The results are given in Table 2.

\subsection{Recording High Efficiency Diffractive Optical Elements} on Flexible Glasses Substrate. This section describes an exploratory study demonstrating successful holographic recording of focusing elements with low spatial frequency and broad working range on flexible glass (Corning(R) Willow(R) Glass) [21]. This novel glass is beneficial for our purpose as it provides better transparency (Table 2) as well as previously mentioned advantages. The flexible glass substrate is dimensionally stable to enable required layer-tolayer alignment, while also displaying low scattering, haze, and absorption. The flexible glass substrate has been demonstrated to be compatible with roll-to-roll and sheet-level coating, lamination, printing processes, as well as stacked multilayer devices with low parallax $[23,28]$.

A holographic lens with an off-axis focusing effect was recorded on Willow Glass of size $26 \times 76 \mathrm{~mm}^{2}$ and $100 \mu \mathrm{m}$ thickness. The layer preparation conditions were identical to the conditions used for the plastic substrate. The diffractive lens element was recorded at $10.5^{\circ}$ away from unslanted position in order to compare the functionality and the performance of the lens element with that recorded on a plastic substrate. Figure 5 shows the angular response of the zero and 1st diffraction order of the recorded lens element. The focal length of the recorded lens element was $6 \mathrm{~cm}$ with diameter of $0.9 \mathrm{~cm}$. It can be observed that a maximum diffraction efficiency of over $90 \%$ was achieved, where 

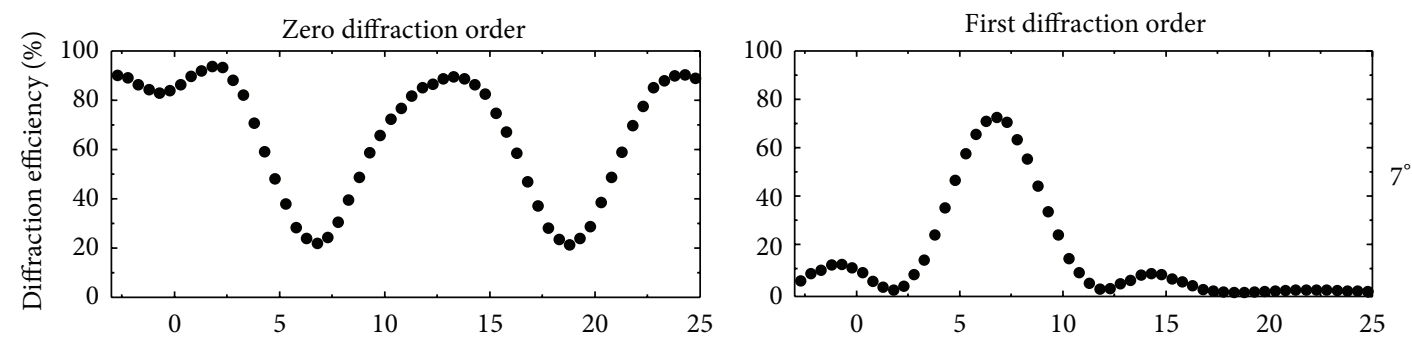

(a)
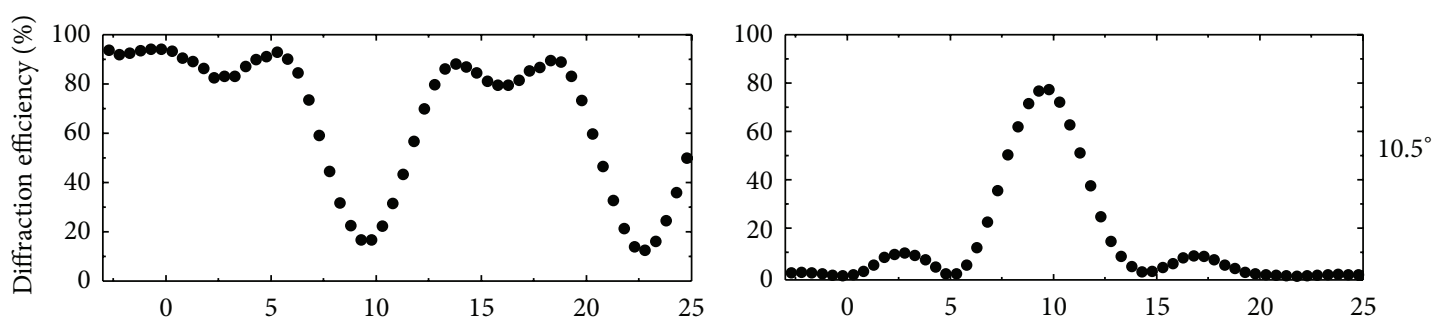

(b)
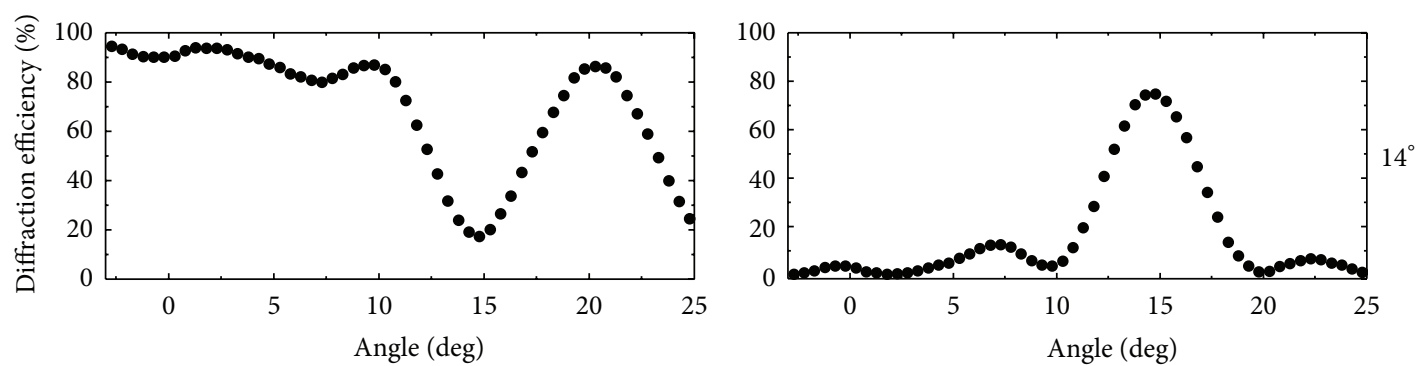

(c)

Figure 3: Angular selectivity curves for diffractive lenses with spatial frequency centred at 300 1/mm recorded in layers with thickness of $50 \mu \mathrm{m}$ on a plastic flexible substrate, at range of angles: (a) $7^{\circ}$, (b) $10.5^{\circ}$, and (c) $14^{\circ}$ before stacking.
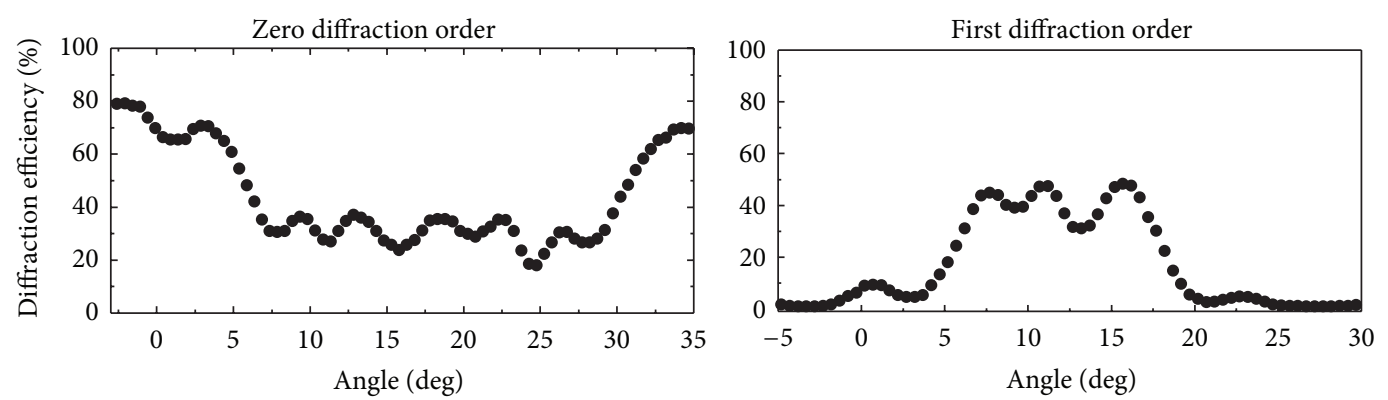

FIGURE 4: Angular selectivity curves for range of combined lens elements recorded with the exposure energy of $60 \mathrm{~mJ} / \mathrm{cm}^{2}$ at spatial frequency of $300 \mathrm{l} / \mathrm{mm}$.

the FWHM was approximately $5^{\circ}$. These very promising results demonstrate that flexible glass is a suitable and stable substrate for holographic recording, while achieving high diffraction efficiency with similar working ranges to the lens elements recorded on plastic substrates.

These encouraging results will prompt new avenues of research into the development of DOEs. The high optical quality of flexible glass (low scattering, birefringence, and absorption) will enable the fabrication of improved devices in any application where device thickness/weight is an issue and where optical losses should be minimized. In addition, the conformable nature of holographic photopolymer devices made on optical glass will enable new device configurations including laminated stacked holographic devices, deformable holographic devices, and combinations of holographic and optoelectronic devices. 

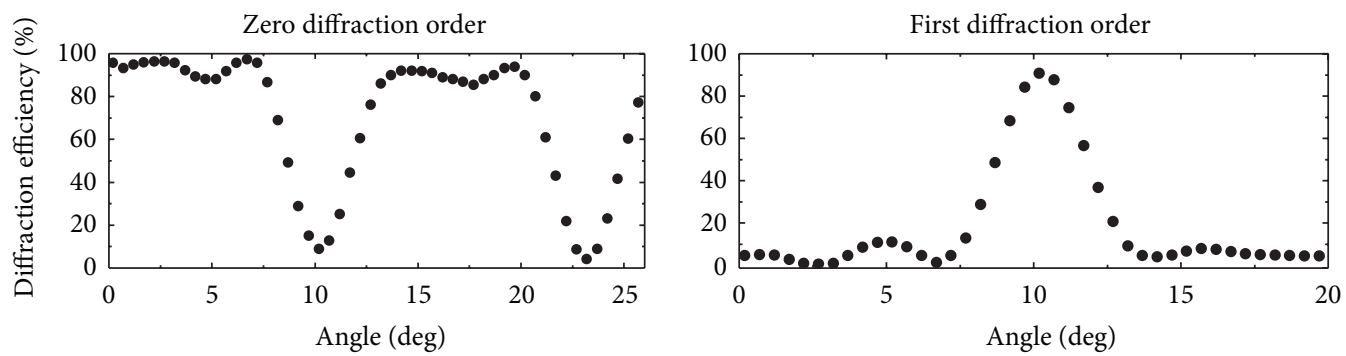

Figure 5: Angular selectivity curves for lens elements recorded at an angle of $10.5^{\circ}$ on flexible glass substrate with the exposure energy of $60 \mathrm{~mJ} / \mathrm{cm}^{2}$ at spatial frequency of $300 \mathrm{l} / \mathrm{mm}$.

Future work will involve recording a series of gratings/lenses on flexible glass and comparison of the loss in diffraction efficiency after stacking the layers with the results above. Investigation into the exploitation of the flexible nature of the glass in order to tune the holographic properties is also currently underway.

\section{Conclusion}

Results confirmed that stacking three holographic elements on plastic substrates increased the acceptance angle of the combined device to $12^{\circ}$ FWHM. The proposed method could be used in applications such as a solar collection and manipulation of beams in illumination systems. For the first time DOEs have been recorded on these flexible glass substrates (Corning(R) Willow(R) Glass). A maximum diffraction efficiency of $90 \%$ was observed in photopolymer layers of $50 \pm 5 \mu \mathrm{m}$ thickness at this spatial frequency. This means that over $90 \%$ of the incident light was measured in the diffracted beam with no correction for reflection, absorption, or other losses. The focal length of the fabricated elements was $6 \mathrm{~cm}$.

Future work will focus on improvement of diffraction efficiency after stacking the layers with large angular response using flexible glass.

\section{Conflict of Interests}

The authors declare that there is no conflict of interests regarding the publication of this paper.

\section{Acknowledgments}

The authors would like to acknowledge the Dublin Institute of Technology, Fiosraigh 2013, for financial support and also the FOCAS Research Institute for providing research facilities. The authors wish to thank Mr. Christopher Smith, Trinity College Dublin, for his advice and support in setting up the collaboration with Corning. Some of these results were presented in Cost 1205 meeting in April 2014.

\section{References}

[1] A. Márquez, C. Neipp, A. Beléndez, S. Gallego, M. Ortuño, and I. Pascual, "Edge-enhanced imaging with polyvinyl alcohol/acrylamide photopolymer gratings," Optics Letters, vol. 28, no. 17, pp. 1510-1512, 2003.
[2] Y. Tomita, K. Furushima, K. Ochi et al., "Organic nanoparticle (hyperbranched polymer)-dispersed photopolymers for volume holographic storage," Applied Physics Letters, vol. 88, no. 7, Article ID 071103, 2006.

[3] A. Pu and D. Psaltis, "High-density recording in photopolymerbased holographic three-dimensional disks," Applied Optics, vol. 35, no. 14, pp. 2389-2398, 1996.

[4] H. Sherif, I. Naydenova, S. Martin, C. McGinn, and V. Toal, "Characterization of an acrylamide-based photopolymer for data storage utilizing holographic angular multiplexing," Journal of Optics A: Pure and Applied Optics, vol. 7, no. 5, pp. 255260, 2005.

[5] I. Naydenova, R. Jallapuram, V. Toal, and S. Martin, "Characterisation of the humidity and temperature responses of a reflection hologram recorded in acrylamide-based photopolymer," Sensors and Actuators, B: Chemical, vol. 139, no. 1, pp. 35-38, 2009.

[6] M. S. Mahmud, I. Naydenova, N. Pandey et al., "Holographic recording in acrylamide photopolymers: thickness limitations," Applied Optics, vol. 48, no. 14, pp. 2642-2648, 2009.

[7] P.-L. Chen, S.-L. Cho, J.-H. Lin, S.-H. Lin, K. Y. Hsu, and S. Chi, "Two-wavelength holographic recording in thick phenanthrenequinone-doped poly(methyl methacrylate) photopolymer," Optical Engineering, vol. 51, no. 3, Article ID 030503, 2012.

[8] K.-F. Yarn, W.-J. Luo, W.-C. Chang, and W.-C. Chuang, "Effects of introducing periodical polymer gratings on the solar cell," International Journal of Physical Sciences, vol. 4, no. 12, pp. 758763, 2009.

[9] H. Akbari, I. Naydenova, and S. Martin, "Using acrylamidebased photopolymers for fabrication of holographic optical elements in solar energy applications," Applied Optics, vol. 53, no. 7, pp. 1343-1353, 2014.

[10] S. Y. Altmeyer, Y. Hu, P. Thiée, J. Matrisch, M. Wallentin, and J. Silbermann, "Multiplexing of transmission holograms in photopolymer," DGaO Proceedings, 2013, http://www.dgao-proceedings.de.

[11] N. Tarjanyi, D. Kacik, D. Sabol, and J. Sheridan, "Low spatial frequency grating recorded in photopolymer material," in Holography: Advances and Modern Trends, vol. 7358 of Proceedings of SPIE, 2009.

[12] I. Pascual, A. Márquez, A. Beléndez, A. Fimia, J. Campos, and M. J. Yzuel, "Copying low spatial frequency diffraction gratings in photopolymer as phase holograms," Journal of Modern Optics, vol. 47, no. 6, pp. 1089-1097, 2000.

[13] S. Martin, C. A. Feely, and V. Toal, "Holographic recording characteristics of an acrylamide-based photopolymer," Applied Optics, vol. 36, no. 22, pp. 5757-5768, 1997. 
[14] T. Babeva, I. Naydenova, D. Mackey, S. Martin, and V. Toal, "Two-way diffusion model for short-exposure holographic grating formation in acrylamidebased photopolymer," Journal of the Optical Society of America B: Optical Physics, vol. 27, no. 2, pp. 197-203, 2010.

[15] I. Naydenova, R. Jallapuram, R. Howard, S. Martin, and V. Toal, "Investigation of the diffusion processes in a self-processing acrylamide-based photopolymer system," Applied Optics, vol. 43, no. 14, pp. 2900-2905, 2004.

[16] S. Gallego, C. Neipp, M. Ortuño, A. Márquez, A. Beléndez, and I. Pascual, "Diffusion-based model to predict the conservation of gratings recorded in poly (vinyl alcohol)-acrylamide photopolymer," Applied Optics, vol. 42, no. 29, pp. 5839-5845, 2003.

[17] S. Martin, P. Leclere, Y. Renotte, V. Toal, and Y. Lion, "Characterisation of an acrylamide-based dry photopolymer holographic recording material," Optical Engineering, vol. 33, no. 12, pp. 3942-3946, 1994.

[18] H. Yao, M. Huang, Z. Chen, L. Hou, and F. Gan, "Optimization of two-monomer-based photopolymer used for holographic recording," Materials Letters, vol. 56, no. 1-2, pp. 3-8, 2002.

[19] R. Jallapuram, I. Naydenova, V. Toal, S. Martin, and R. Howard, "Spatial frequency response of acrylamide holographic photopolymer," in Proceedings of the International Conference on Laser Applications and Optical Metrology, C. Shakher and D. S. Mehta, Eds., pp. 275-279, Anamaya Publishers, New Delhi, India, 2003.

[20] H. Kogelnik, "Coupled wave theory for thick hologram gratings," Bell System Technical Journal, vol. 48, no. 9, pp. 2909-2947, 1969.

[21] S. Garner, S. Glaesemann, and X. Li, "Ultra-slim flexible glass for roll-to-roll electronic device fabrication," Applied Physics A, vol. 116, no. 2, pp. 403-407, 2014.

[22] D. A. Mourey, R. L. Hoffman, S. M. Garner et al., "Amorphous oxide transistor electrokinetic reflective display on flexible glass," in Proceedings of the 18th International Display Workshops (IDW '11), pp. 1309-1312, Nogoya, Argentina, December 2011.

[23] S. Garner, G. Merz, J. Tosch et al., "Ultra-slim flexible glass for electronic application," in Proceedings of the MRS Fall Meeting, Boston, Mass, USA, November 2012.

[24] E. Hecht, Optics, Addison-Wesley, 3rd edition, 1998.

[25] J. T. Gallo and C. M. Verber, "Model for the effects of material shrinkage on volume holograms," Applied Optics, vol. 33, no. 29, pp. 6797-6806, 1994.

[26] V. Toal, Introduction to Holography, CRC Press, Taylor \& Francis Group, Boca Raton, Fla, USA, 2011.

[27] D. Cody, E. Mihaylova, L. O’Neill et al., “Effect of zeolite nanoparticles on the optical properties of diacetone acrylamide-based photopolymer," Optical Materials, vol. 37, pp. 181-187, 2014.

[28] S. Garner, G. Merz, G. S. Glaesemann et al., "26.1: invited paper: ultra-slim flexible glass substrates for display applications," SID Symposium Digest of Technical Papers, vol. 43, no. 1, pp. 342-344, 2012. 

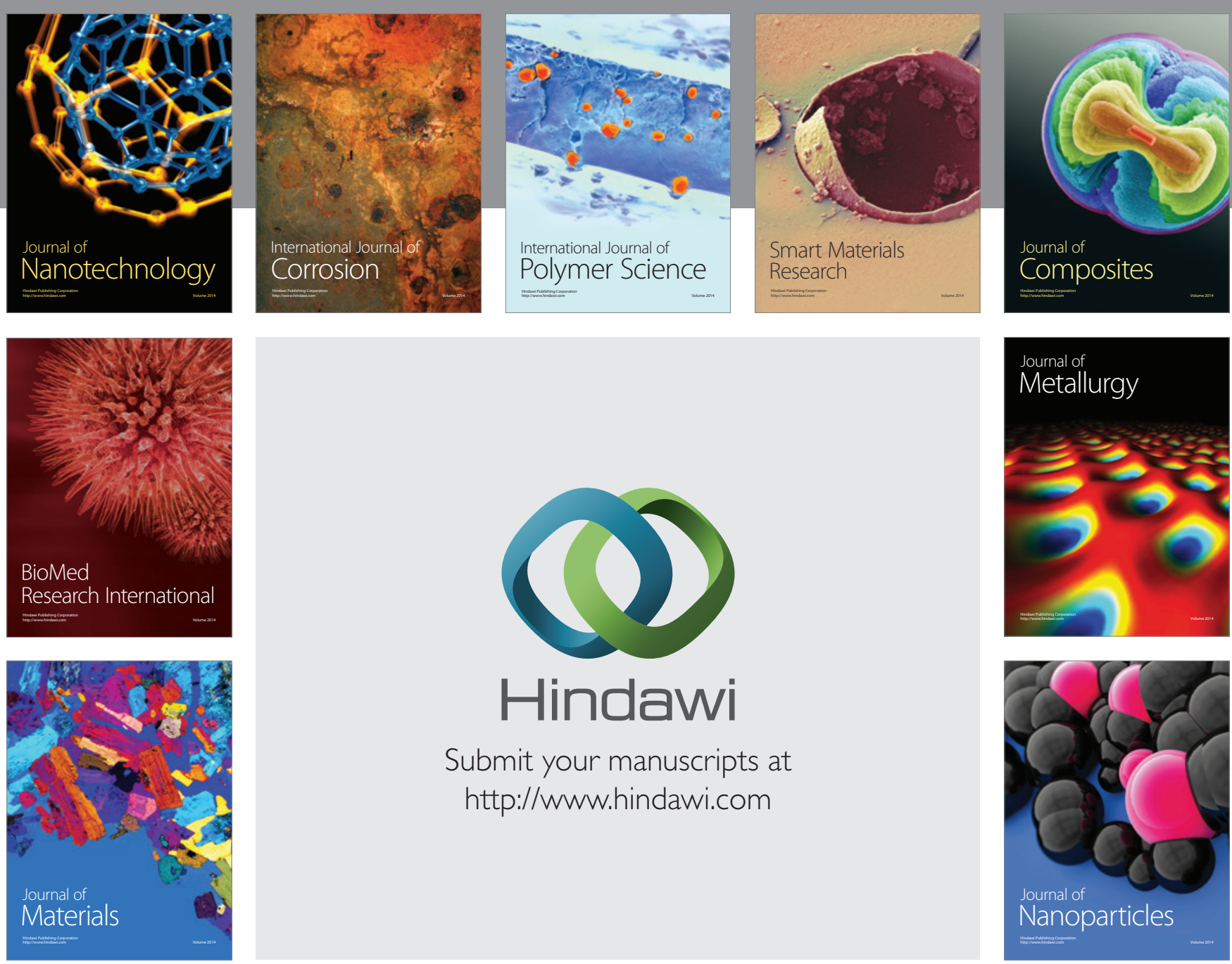

Submit your manuscripts at http://www.hindawi.com
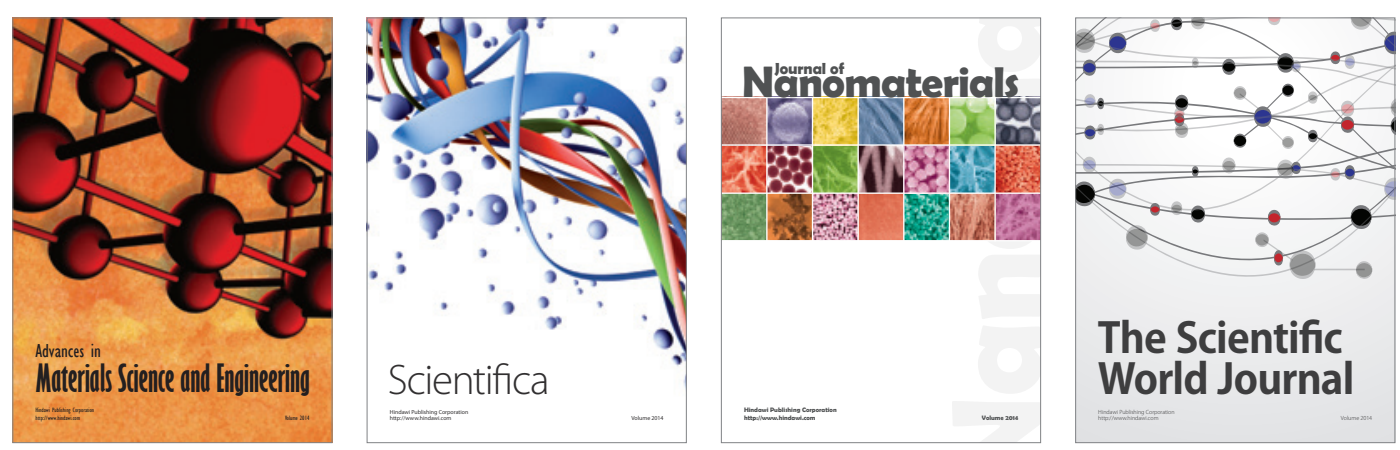

\section{The Scientific World Journal}
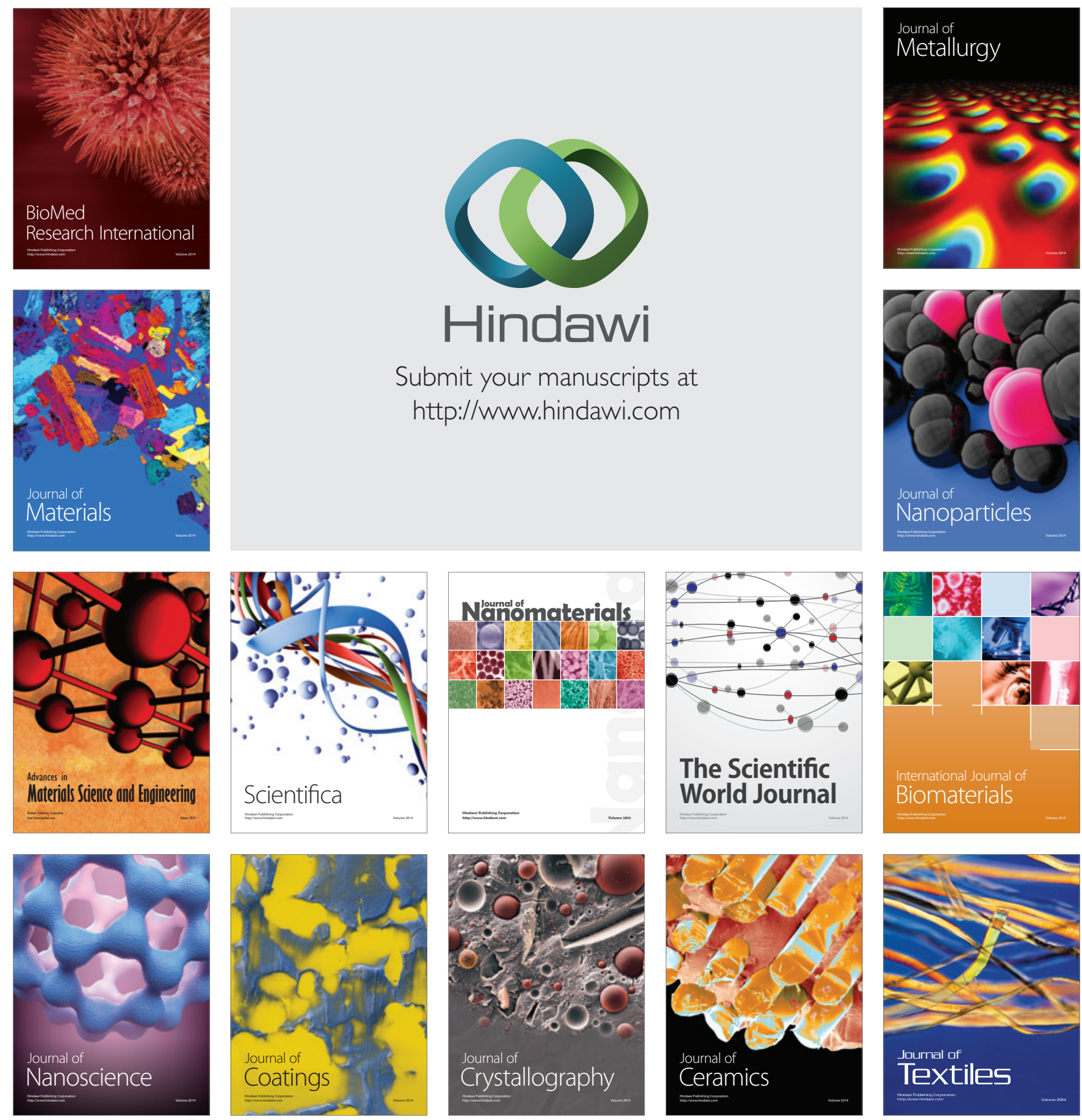